\section{Giving up the big questions when answers are in sight}

SIR - Your Editorial and News Feature on the James Webb Space Telescope (JWST) correctly point out that large missions are difficult for NASA to accommodate during times of constrained budgets (Nature 440, 127 and $140-143 ; 2006)$. But you do not explore what the community gives up if the largest missions such as JWST are sacrificed or delayed.

These 'flagship' missions are expensive because they are not merely large but scientifically transformational. One of the most intellectually significant, and humbling, scientific discoveries of our lifetime is that the atoms and forces of which we and our environment are made and that hold us together are but a trace contaminant of the true composition of the Universe, which is 96\% dark matter and darkenergy. These and similar discoveries simply could not have been made by a large number of low-cost space-science missions.

JWST has similar paradigm-breaking capabilities, in fields as diverse as the search for life-forming mechanisms on extra-solar planets and the birth of galaxies. In the era when it is scheduled to fly, all the current great observatories (Hubble, Chandra and Spitzer) will have completed their missions, or nearly so. A decision to give up on a small number of flagship missions is not merely a decision of economics and resource distribution, but a fundamental decision to avoid solving the grandest problems of science at a time when we know that they are within our reach.

\section{Matt Mountain}

Space Telescope Science Institute, 3700 San Martin Drive, Baltimore, Maryland 21218, USA

\section{Too many people are being displaced for Merowe dam}

SIR - Your Special Report ${ }^{\alpha}$ Tide of censure for African dams" (Nature 440, 393-394; 2006) considers the social consequences of the Merowe project in Sudan, which will displace up to 50,000 people. Can projects that involve such large population transfers possiblybe justified?

The principal argument in favour of the Merowe project is a pressing need for electricity in Sudan. Merowe will provide electrical power corresponding to an average load of $625 \mathrm{MW}$ (see en.wikipedia.org/wiki/ Merowe_Dam).

With 50,000 displaced people for $625 \mathrm{MW}$ of electricity, what I call the 'displacement index' for Merowe is 80 people per MW. By comparison, the smaller dam at Tignes in the French Alps, whose construction in 1953 caused agitation and rebellion, displaced 384 people, for an average load of $103 \mathrm{MW}$. The corresponding 'displacement index' is 3.7 one-twentieth that of Merowe.

This discrepancy is all the more sinister when one takes into account that more than 50 years have elapsed since Tignes. In the interval, the human suffering generated by resettlement schemes has, presumably, received increased recognition and respect.

In this time, friendlier power generating alternatives have also proven their merit. It is significant that a single present-day nuclear reactor (with an average load of $960 \mathrm{MW}$ ) would provide more power than the Merowe dam, with the displacement of very few people, if any. Would that not be the greener solution? Maurice Guéron

15 rue Lakanal, 75015 Paris, France

\section{Can the Internet save us from epidemics?}

SIR - Kathleen Morrison, in News \& Views ("Failure and how to avoid it" Nature 440, $752-754 ; 2006$ ), notes that societies have often prevented collapse by adopting new technological strategies. In today's world, where one of the most-talked about prospects for collapse is an epidemic of infectious disease, it is worth remembering that perhaps we already have the technological strategy to avoid it - the Internet.

Remote working, made possible by the Internet ('telepresence'), is already a key component of national and business pandemic plans. Telepresence can inhibit viral transmission by reducing human-tohuman contact. Prepared organizations can leverage telepresence to allow continued productivity and functioning of supply chains during an outbreak.

Past societies often reacted to epidemics by bunching together, increasing density and transmission rates. In medieval Europe, for example, warring religious factions demonstrated solidarity in the face of a plague by marching together in the streets. And Native Americans expressed goodwill by gathering in the teepees of those infected with smallpox. But if we are well-prepared when an epidemic arrives, we can fluidly shift into a self-quarantined, telepresent society in which microbes fail by dint of host sparseness.

Whatever the social ills of increased isolation in our computer age, they may bode worse for the microbes than for us.

David M. Eagleman

Department of Neurobiology and

Anatomy, University of Texas, Houston,

Texas 77030, USA

\section{Plant databases linked for botanists and gardeners}

\section{SIR - Your Editorial ${ }^{\alpha}$ The constant} gardeners ${ }^{\prime}$ (Nature 440,$845 ; 2006$ ) calls for closer cooperation between botanical gardens to integrate information about plants and make it accessible to all botanists. One such project, PlantCollections, is already under way. PlantCollections links the databases of 16 botanical gardens across the United States, using distributed queries on the Web. This approach allows organizations to keep their own database formats while sharing information. It uses software that translates an Internet query into the unique formats used by each of the 16 botanical gardens, collates the results and presents them to the user as a single document. The application is inexpensive to deploy and easy to maintain, so additional gardens can be added with minimal costs.

Information about living plant collections, herbarium specimens, long-term stored seeds, DNA samples and image holdings within each institution is shared in PlantCollections in a format that everyday gardeners as well as research scientists can understand. A list of participating botanical gardens and arboreta will be available at www.publicgardens.org. Further information on joining the project can be obtained from the author.

\section{Boyce Tankersley}

Chicago Botanic Garden, 1000 Lake Cook Road, Glencoe, Illinois 60022, USA

\section{People of the past should not be called primitive}

\section{SIR - Your Editorial "Rightful owners"} (Nature 440,716; 2006) and Special Report “Guinea experts cry foul on tribal exhibits" (Nature 440, 722-723;2006) help bring to light the importance and challenges of repatriation and reclamation. But in them, you describe the makers of these antique South Pacific artefacts as "primitive": a term that has been recognized as inappropriate by anthropologists for decades.

Referring to the communities that made this art as "primitive" implies that they were somehow beneath Western societies. Cultures developed uniquely across the world - these differences do not make one society better than another.

The word "primitive" reinforces colonial ideas of the innate rights of western societies to control others, and maintain their economic and cultural hold over these groups today. Megan Marie McCullen

Department of Anthropology, Michigan State University, 354 Baker Hall, East Lansing, Michigan 48824, USA 